\title{
Efficient Treatment of Fish Intestinal Parasites Applying Biocompatible Membrane-penetrating Oral Drug Delivery Nanoparticles
}

\section{Patrick D. Mathews}

Federal University of Sao Paulo (UNIFESP)

Ana C.M.F. Patta

Federal University of Sao Paulo (UNIFESP)

Rafael R.M. Madrid

Federal University of Sao Paulo (UNIFESP)

Carlos A.B. Ramirez

Federal University of Sao Paulo (UNIFESP)

Omar Mertins ( $\nabla$ mertins@unifesp.br)

Federal University of Sao Paulo (UNIFESP)

\section{Research Article}

Keywords: Nanoparticle, Biomaterial, Nanomedicine, Gastrointestinal tract, Biological membrane

Posted Date: April 13th, 2021

DOl: https://doi.org/10.21203/rs.3.rs-403291/v1

License: (c) (1) This work is licensed under a Creative Commons Attribution 4.0 International License. Read Full License

Version of Record: A version of this preprint was published at ACS Biomaterials Science \& Engineering on November 15th, 2021. See the published version at https://doi.org/10.1021/acsbiomaterials.1c00890. 


\title{
Efficient treatment of fish intestinal parasites applying biocompatible membrane-penetrating oral drug delivery nanoparticles
}

\author{
Patrick D. Mathews*, Ana C.M.F. Patta, Rafael R.M. Madrid, Carlos A.B. Ramirez, Omar \\ Mertins* \\ Laboratory of Nano Bio Materials (LNBM), Department of Biophysics, Paulista Medical School, \\ Federal University of Sao Paulo (UNIFESP), 04023-062 Sao Paulo, Brazil \\ *E-mail addresses: \\ patrick.mathews@unifesp.br (P.D. Mathews); mertins@unifesp.br (O. Mertins)
}

\begin{abstract}
Nanodelivery of drugs aims to ensure drug stability in the face of adverse biochemical conditions in the course of administration, concomitant with appropriate pharmacological action provided by delivery at the targeted site. In this study, the application potential of nanoparticles produced with biopolymers chitosan- $N$-arginine and alginate as an oral drug delivery material is evaluated. Being both macromolecules weak polyelectrolytes, the bioparticle presents strong thermodynamic interaction with a biological model membrane consisting of charged lipid liposome bilayer, leading to membrane disruption and membrane penetration of the bioparticles in ideal conditions of $\mathrm{pH}$ corresponding to the oral route. The powder form of the bioparticle was obtained by lyophilization and with a high percentage of entrapment of the anthelmintic drug praziquantel. In vivo studies were conducted with oral administration to Corydoras schwartzi fish with high intensity of intestinal parasites infection. The in vivo experiments confirmed the mucoadhesive and revealed membrane-penetrating properties of the bioparticle by translocating the parasite cyst, which provided target drug release and reduction of over $97 \%$ of the fish intestinal parasites. Thus, it was evidenced that the bioparticle was effective in transporting and releasing the drug to the target, providing an efficient treatment.
\end{abstract}

Keywords: Nanoparticle; Biomaterial; Nanomedicine; Gastrointestinal tract; Biological membrane. 


\section{Introduction}

The oral administration of drugs is preferred in human and veterinary medicine thanks to many practical benefits as high compliance, better disease management, reduced costs, easy of distribution and selling in the markets [1]. However, this route o administration is often not possible due to plethora of drawbacks related to drugs stability and degradability, besides toxicity and poor bioavailability [2,3]. The drugs administration-complexity of oral route is related besides to the extreme $\mathrm{pH}$ variation of the gastrointestinal tract, specially between stomach and intestine, which requires for instance drugs stability in strong acid and then favored absorption properties in the intestine. In this context, drug carriers such as nanoparticles and submicrometric devices have become a technological means to potentialize oral drug administrations by overcoming the main related problems, as mentioned above, and besides improving the drugs absorption and effective action, as well as reducing related side effects [4-9].

Currently, it is well recognized that the best development of drug carriers starts from adequate characterization of the physical-chemical properties and from evaluation of the interaction with biological medium of application interest. Furthermore, the efficiency in drug delivery of nanoparticles can be associated to the uptake by the targeted cells, with transport through the plasmatic membrane. Thus, membrane-penetrating nanoparticles may provide advantageous application properties for improved targeted drug delivery [10-14].

In the gastrointestinal tract, endocytose has been described as the major mechanism of absorption of nanoparticles by translocating the intestinal lymph node (Peyer's patches) $[15,16]$. Besides, many studies have reported that the distribution of nanoparticles in the intestinal cavity was in the apical region of $M$ cells, where the same were engulfed by endocytosis $[17,18]$. Comparing with normal epithelial cells, it is known that $M$ cells reduce the activity of membrane hydrolase affecting the uptake of protein or protein-modified nanoparticles $[18,19]$. It has been further shown that nanoparticles with mucoadhesive properties can change the permeability of mucous epithelium and improve the bioavailability of protein and peptide drugs [19]. Indeed, mucosal adhesion of nanoparticles may prolong the retention time in gastrointestinal tract, hence enhancing membrane-penetrating processes [20]. Chitosan and chitosan-derivatives based nanoparticles are well recognized as promising drug delivery materials due to the inherent characteristic of chitosan in providing mucoadhesion influenced by electrostatic interactions and hydrophilic/hydrophobic contributions, which by turn may contribute in biological membrane translocation [21-25]. For instance, Yeh et al. [26] showed that chitosan has the capability to open the tight junction of Caco 2 monolayer cells, thus reducing its transmembrane resistance and increasing the permeability of the cell. Nevertheless, the practical application of nanoparticles as oral drug delivery devices relays besides on challenges associated to the short turnover cycle of mucus in the intestine, which by turn eliminates the drug carriers, consequently reducing the drug bioavailability $[10,27]$. Thereby, efficient muco and membrane-penetrating nanoparticles 
represent promising materials to real application and throughput in oral drug delivery technologies.

In this study, we aimed at improving the physicochemical and biological interaction studies of a nano-submicrometric bioparticle as an oral drug carrier, produced with chitosan- $N$-arginine and alginate, both biocompatible, biodegradable polysaccharides and opposite charged polyelectrolytes which provide mucoadhesiveness to intestinal mucosa [28]. Chitosan was covalently linked to arginine, an $\alpha$-amino acid with essential functions in several biochemical processes and of cell penetrating characteristics [14]. With a pKa of 13.8 [29], its guanidinium group is positively charged in neutral, acidic and alkaline media. The bioparticles colloidal structure, surface charge and thermodynamic interactions with a lipid model membrane were studied in $\mathrm{pH}$ conditions covering the oral administration route. Furthermore, the anthelmintic drug praziquantel was entrapped and in vivo studies were performed by oral administration of the lyophilized drug carrier to Corydoras schwartzi fish as animal model $[8,28,30]$, highly infected with digenetic trematode parasites in the intestinal tract. Therefore, the study aimed at approaching the application of the bioparticles by evaluating the effective energetics in ideal conditions, and as well the in vivo effectiveness of drug delivery, promoted by membrane-penetrating characteristics.

\section{Materials and methods}

\subsection{Materials}

Chitosan- $N$-arginine was obtained, purified and characterized as previously described (Patta et al., 2020) containing 95\% degree of deacetylation (DDA), average molecular weight (Mw) $135 \mathrm{kDa}$, low viscosity ( $<20 \mathrm{cP}$ ) and 3.5\% of monomers chemically bonded to arginine. Sodium alginate (from brown algae Macrocystis pyrifera) was from Sigma-Aldrich (St. Louis, MO, USA) with $61 \%$ mannuronic acid and 39\% guluronic acid with an average $\mathrm{Mw}=200 \mathrm{kDa}$ (1143 uronic acid units) and low viscosity (5-40 cP). Alginate main solution was prepared by overnight stirring in acetate buffer $(80 \mathrm{mM}, \mathrm{pH} 4.50 \pm 0.02)$ at $1 \mathrm{mg} / \mathrm{mL}$ and diluted when required. The lipid 1-palmitoyl-2-oleoyl-sn-glycero-3-phospho-(1'-rac-glycerol) (POPG) from Avanti Polar Lipids (99\%) in powder form was dissolved in chloroform and kept at $-20{ }^{\circ} \mathrm{C}$ until use. Praziquantel (2-(cyclohexylcarbonyl)-1,2,3,6,7-11b-hexahydro-4H-pyrazino[2,1-a] isoquinolin -4-one; 98\%) and fluorescein isothiocyanate isomer I (FITC; 90\%) were from Sigma-Aldrich. All other reagents were of analytical grade. Solutions were prepared using deionized water from the Milli-Q Millipore system with a total organic carbon value of $<15 \mathrm{ppb}$ and a resistivity of $18 \mathrm{M} \Omega \mathrm{cm}$. The molecular structures of the drug and biopolymers are shown in Figure 1.

\subsection{Production of bioparticles}

The lyophilized powder of chitosan- $N$-arginine ( $\mathrm{CHarg}$ ) was dissolved in acetate buffer ( 80 $\mathrm{mM}, \mathrm{pH} 4.50 \pm 0.02$ ) at $1 \mathrm{mg} / \mathrm{mL}$ by vortex mixing for $3 \mathrm{~min}$ then submitted to bath sonication $\left(40 \mathrm{kHz}, 25^{\circ} \mathrm{C}\right.$; Eco-Sonics Q3.0L, Brazil) for $30 \mathrm{~min}$. The pre-dissolved alginate solution was diluted to $0.1 \mathrm{mg} / \mathrm{mL}$ with the same buffer. Both solutions were filtered using $0.8 \mu \mathrm{m}$ pore size 
filters (Millipore). Typically, $50 \mathrm{~mL}$ of alginate solution were titrated at $2 \mathrm{~mL} / \mathrm{min}$ into $50 \mathrm{~mL}$ of CHarg solution under stirring $(1200 \mathrm{rpm})$ and after the mixture was kept under stirring overnight at $22 \pm 2{ }^{\circ} \mathrm{C}$. In this way the colloidal bioparticles were obtained with a strong electrostatic interaction between the two oppose charged macromolecules [28]. For the production of the drug-containing particles, praziquantel was pre-interacted with $\mathrm{CHarg}$ by previous inclusion of $38 \mu \mathrm{M}$ in the $\mathrm{CHarg}$ solution then submitted to overnight stirring at 800 $\mathrm{rpm}$ and next the above protocol was followed. For the studies of different $\mathrm{pH}$ conditions, an aliquot of $100 \mu \mathrm{L}$ was diluted to $10 \mathrm{~mL}$ in different buffers. The buffer of $\mathrm{pH} 2.50$ was prepared using sodium citrate and hydrochloric acid; buffer of $\mathrm{pH} 4.50$ and 6.50 were prepared with sodium acetate and acetic acid by varying the proportions of salt and acid to achieve the desired $\mathrm{pH}$; and buffer of $\mathrm{pH} 7.80$ was prepared with potassium dihydrogen phosphate and disodium phosphate. The maximal variation in $\mathrm{pH}$ was 0.02 and all buffers were at the same concentration of $8 \mathrm{mM}$.

In order to obtain the bioparticles in powder form for oral administration, the colloidal dispersions were freeze-dried (Liotop, Liobras, Brazil) during $48 \mathrm{~h}$ and then saved in a refrigerator at $4{ }^{\circ} \mathrm{C}$ until use.

An additional step in preparation was included for the production of the particles containing the fluorescent probe FITC, which was dissolved in methanol at $0.5 \mathrm{mg} / \mathrm{mL}$ and $100 \mathrm{~mL}$ of this solution was slowly mixed with the same volume of particles solution. The mixture was kept under stirring at $800 \mathrm{rpm}$ overnight then submitted to water bath at $70{ }^{\circ} \mathrm{C}$ during $5 \mathrm{~h}$ under stirring at $1200 \mathrm{rpm}$ in order to evaporate the methanol. Finally, the above procedure was followed and the samples were equally lyophilized.

\subsection{Determination of drug entrapment}

As a standard procedure to obtain the percentage of drug effectively incorporated into the bioparticles, a standard curve was initially constructed by measuring the absorbance of different concentrations of praziquantel with a spectrophotometer (Ultrospec UV-Visible GE 8000 ) in clean quartz cuvettes. Concentrations from 1.8 to $38 \mu \mathrm{M}$ were measured, representing from 5 to $100 \%$ of the drug concentration in the bioparticle. The drug was dissolved in a solution of methanol and water $(60: 40, v: v)$ due to the impossibility of dissolving in pure water. The absorbance readings were taken at a wavelength of $263 \mathrm{~nm}$. To obtain the data, the software Datrys Life Science was used. To calculate the percentage of drug entrapment, after construction of the calibration curve, the absorbance of the solution of the bioparticles containing the drug was also measured. For this, the lyophilized powder of bioparticles containing $38 \mu \mathrm{M}$ of praziquantel was dispersed in $3 \mathrm{~mL}$ of water under stirring for $3 \mathrm{~min}$ and then centrifuged (HITACHI Himac CT15E) at $3500 \mathrm{rpm}$ for $4 \mathrm{~min}$ at $25^{\circ} \mathrm{C}$. The bioparticle-free supernatant was collected with a micropipette and mixed with methanol in the same proportion as the solutions used for the pure drug. The absorbance of the supernatant was also measured. The percentage of drug entrapment was obtained by extrapolating the calibration curve and comparing it with the absorbance of the supernatant. 


\subsection{Production of model membrane}

Liposomes were produced as a biological membrane model using $200 \mu \mathrm{L}$ of a POPG lipid solution at $43.12 \mathrm{mM}$ in chloroform, which was evaporated with nitrogen gas, creating a lipid film in the falcon tube where it was later hydrated with acetate buffer $\mathrm{pH} 4.50$, maintained in vortex for 3 minutes and 3 minutes under ultrasonic tip with a frequency of $20 \mathrm{kHz}$. Amounts of $120 \mu \mathrm{L}$ of liposomes were further diluted to $2 \mathrm{~mL}$ with the different buffer solutions as described for the bioparticles and sonicated once more. The preparation of the vesicles was based on previous studies [31].

\subsection{Transmission electron microscopy}

After preparation of the colloidal dispersions, a portion of concentrated bioparticles was centrifuged for $3 \mathrm{~min}$ at $900 \mathrm{rpm}$. The supernatant was discharged and bioparticles pellets were resuspended in $100 \mu \mathrm{L}$ of buffered formaldehyde $2 \%$. Then, drops of $20 \mu \mathrm{L}$ of samples were placed on film in a flat surface for its adsorption to formvar coated nickel grids (on-gridmethod). After $15 \mathrm{~min}$, grids were gently washed with $0.1 \mathrm{M}$ sodium cacodylate buffer. Samples were negatively stained with saturated uranyl acetate for $10 \mathrm{~min}$, then uranyl excess was blotted on filter paper. Grids were dried right before the observation, which was performed at $80 \mathrm{kV}$ under a JEOL 1200 EX II transmission electron microscope. Micrographs were captured with a GATAN 791 camera.

\subsection{Dynamic light scattering (DLS) and zeta potential}

The hydrodynamic diameters of the bioparticles in aqueous suspensions were analyzed by DLS with a Malvern Zetasizer $300 \mathrm{ZS}$ (Malvern Instruments, UK), operating with a $4 \mathrm{~mW}$ HeNe laser at a wavelength of $632.8 \mathrm{~nm}$ and detection at the angle of $173^{\circ}$. The measurements were performed in a temperature-controlled chamber at $25{ }^{\circ} \mathrm{C}$. The typical autocorrelation functions were acquired using exponential spacing of the correlation time and data analyses were performed with software provided by Malvern. The intensity-weighted size distribution was acquired by fitting data with a discrete Laplace inversion routine and the size determination was accomplished using Stokes-Einstein relation [32]. The polydispersity was obtained with second-order cumulant analysis of the correlation functions applying the amplitude of the correlation function and the relaxation frequency. The zeta potential of the bioparticles was obtained with the same Malvern Zetasizer, in acquiring at least 100 runs per sample at $25{ }^{\circ} \mathrm{C}$. The principle of the measurement is based on laser Doppler velocimetry, where the electrophoretic mobility $u$ is converted to zeta potential $\zeta$ by applying the Helmholtz Smoluchowski relation: $\zeta=$ u $/ \varepsilon \varepsilon_{0}$, where $\eta$ is the solution viscosity, $\varepsilon$ the dielectric constant of water, and $\varepsilon_{0}$ the permittivity of free space. In both experiments, folded capillary cells were employed.

\subsection{Isothermal titration calorimetry (ITC)}

ITC measurements were performed in a VP-ITC microcalorimeter (MicroCal Inc., Northampton, MA). Before the measurements, all samples were degassed for 5 min under 
reduced pressure in order to avoid the interference of air bubbles in the aqueous suspensions. Following the standard procedure, the working cell of $1.442 \mathrm{~mL}$ in volume was filled with the liposome dispersion in the specific buffer solution. One aliquot of $2 \mu \mathrm{L}$ followed by 27 aliquots of $10 \mu \mathrm{L}$ of the bioparticle suspension in the same buffer were injected stepwise with a $300 \mathrm{~s}$ interval into the working cell. The sample cell was constantly stirred (307 rpm), and the experiments were performed at $25^{\circ} \mathrm{C}$. The data acquisition and analyses were carried out with Origin software provided by MicroCal. The data fitting was performed applying the single set of identical binding sites model, as previously described [31].

\subsection{In vivo experiments}

For in vivo experiments 180 adult specimens of Corydoras schwartzi fish, bought from commercial provider, were employed following procedures of our previous studies $[8,28,30]$. The fish were divided in three aquariums with $15 \mathrm{~L}$ of dechlorinated water, previously conditioned to adequate water conditions for the species, with a constant temperature of 28 ${ }^{\circ} \mathrm{C}$ using thermostat systems (Hopar Aquarium Heater H-606 150W), constant pH 6.8 and adjusted physicochemical parameters (Seachem Prime water conditioner) and submitted to constant filtration and aeration (Aquatech FE25 Filtration System) with the water flow rate adjusted to $250 \mathrm{~L} / \mathrm{h}$. The fish were nourished with commercial ration TetraMin (Tetra $\mathrm{GmbH}$, Germany), adequate for tropical ornamental fish, twice a day (8 AM and 6 PM) and acclimated for ten days before experiments. A $12 \mathrm{~h}$ light/dark cycle was adopted in the aquariums room. The fish were kept in $14 \mathrm{~h}$ fasting before the experiments. The first aquarium was destinated as the first control, with fish kept with normal ration feeding; the second aquarium was the control for the administration of bioparticles free of drug; and the third for bioparticles containing praziquantel. To the second and third aquariums, $400 \mathrm{mg}$ of lyophilized bioparticles containing fluorescent probe FITC were dispersed in each one and the filtration systems were turned off during $20 \mathrm{~min}$ in order to avoid particles retention. All fish avidly fed on particles. This procedure was performed twice a day during three days and $30 \mathrm{~min}$ before the normal nourishing. The administration procedure provided a total of $24.2 \mathrm{mg}$ of praziquantel per fish $\mathrm{kg}$ body weight ( $\mathrm{mg} / \mathrm{kg}$ b.w.) divided in the six doses. The average fish b.w. was obtained by weighting all fish from the third aquarium during the course of the experiment. From the fourth day on, only normal ration feeding was provided. After 2, 6 and $24 \mathrm{~h}$ of the first bioparticles administration, three individuals of each aquarium were sacrificed for initial intestinal examination. In the typical procedure, fish were individually collected with an appropriate collecting net and immediately euthanized by neural pithing [33]. In necropsies the intestines were removed, opened longitudinally, placed on microscopy slides, rinsed with MilliQ water using a micropipette and readily examined in a Confocal Leica TCS SP8 Inverted Microscope, using 10x, 40x, 63x and 100x objectives, where the fluorescent dye FITC was excited with a diode-pumped solid-state laser at $480 \mathrm{~nm}$ and the emission signal was collected at $525 \mathrm{~nm}$. The Leica Application Suite $X$ was used for image capture and analysis.

For the intestinal parasites survey, ten individuals from the first control aquarium where equally examined, following the procedures described above, for parasites total counting in a 
Primo Star Zeiss Microscope under bright field using 10x objective. Equally, ten individuals from the second and ten from the third aquariums were so examined after every $7,14,21,28$ and 35 days from the first administration of bioparticles.

The parasitic indices of prevalence $(\mathrm{P})$ and mean intensity (MI) were calculated according to the criteria by Bush et al. [34]:

Prevalence (\%):

$\mathrm{P}=\mathrm{Np} / \mathrm{NE} \times 100$

where $\mathrm{Np}$ is the number of fish infected by intestinal parasites and NE is the total number of fish examined.

Mean intensity (MI):

$\mathrm{MI}=\mathrm{Nsp} 1 / \mathrm{NPsp} 1$

where Nsp1 is the number of a given class of parasite and NPsp1 is the number of fish infected by a given class of parasite.

The study was carried out in compliance with the ARRIVE guidelines and the protocols were approved by the Animal Ethical Committee of the Federal University of Sao Paulo and all experiments were performed in accordance with the U.K. Animals (Scientific Procedures) Act, 1986 and associated guidelines, EU Directive 2010/63/EU for animal experiments, and the National Institute of Health guide for the care and use of Laboratory animals (NIH Publications No. 8023, revised 1978).

\section{Results and discussion}

\subsection{Bioparticles morphology}

Figure 2 shows TEM photomicrographs of the bioparticles evidencing an overall random coiled structure, which is well in accordance to the theory for the assembling of polyelectrolytes of opposite charges in solution [35]. Figure $\mathbf{2} \mathbf{b}$ shows a particle which is representative to the so called "scrambled egg" coexisting with "latter" structure [36], i.e., with a concentrated central region of about $200 \mathrm{~nm}$ in diameter and containing random branches of polymer chains hanging away from the surface. The actual structure can thus be represented as an intermediate random coil/swollen gel, combining a mainly hydrophobic core, produced by the partial charge neutralization occurred with the interaction between the oppose charged polyelectrolytes, along with hydrophobic ladder-like segments coexisting with disordered hydrophilic segments hanging from the central region. The extension and characteristics of these branches on the surface of the particles were discussed in our previous study in analyzing DLS, zeta potential and SAXS data in different $\mathrm{pH}$ conditions [28]. It was evidenced that the extension and conformation of these branches is strongly dependent on $\mathrm{pH}$. Figure $\mathbf{2} \mathbf{c}$ and $\mathbf{d}$ show a typic aggregate of the same bioparticles after resuspension in a same buffer solution of $\mathrm{pH} 4.50$, but of lower salt concentration of $8 \mathrm{mM}$. The result shows the influence of the ionic strength on the bioparticle physical characteristics, further discussed in the following section. 


\subsection{Interaction with lipid membrane}

\subsubsection{Colloidal structuration}

Drug delivery devices designed as membrane-penetrating nanoparticles are expected to stablish strong physicochemical interactions with the targeted membrane. In order to estimate the dimension of this interaction, a calorimetric study was performed aiming to evaluate the thermodynamic interaction of the bioparticle with a model of lipid membrane. Since lipids bilayer consists the main supporting structure of cell membranes, one aimed to analyze the energetic intensities when the mucoadhesive bioparticle comes in physical contact with negative charged lipids of a bilayer. Considering oral administration, the main question here was the $\mathrm{pH}$ dependency, which could be helpful for revealing the energetics involved in the interaction with a lipid membrane in each $\mathrm{pH}$ condition.

For this specific survey, the bioparticle was prepared as previously described, but instead of buffer at $80 \mathrm{mM}$ the particle was dispersed in the new buffers of concentrations $8 \mathrm{mM}$. The reduced ionic strength of the solution actually produced a significant impact in the structure and surface charge of the bioparticle. Indeed, at $80 \mathrm{mM}$ the bioparticle pH-dependence is expressive considering both hydrodynamic diameter and zeta potential, as we previously reported [28]. However, in $8 \mathrm{mM}$, the $\mathrm{pH}$ dependence almost vanished, with diameters roughly around $400 \mathrm{~nm}$ and large distribution and slightly positive zeta potentials in all pHs (Table 1). An exception is given for size in $\mathrm{pH} 4.50$, where additionally larger aggregates were produced leading to a bimodal size distribution.

However, the low ionic strength condition was preferred for the ITC study in order to minimize ionic charges screening effects on chitosan [37,38], which could interfere in an adequate evaluation of the effective thermodynamic interaction between the particles and the lipid bilayer.

As a standard procedure, DLS and zeta potential experiments were performed with the bioparticles and liposomes in the different buffers before the same samples were introduced in the ITC equipment, and the same measurements were performed again with the samples resulting from ITC. As shown in Table 1, similar to the bioparticles, the POPG liposomes presented almost even hydrodynamic diameter and zeta potential for all pH conditions. Despite, a remark is given to the large zeta potential standard deviation, which may be related to repulsive effect between the liposomes of strong negative surface charge, leading to variations in the electrophoretic mobility during zeta potential measurements.

However, remarkable results were found after the titration of bioparticles into dispersions of negative charged liposomes. In $\mathrm{pH} 2.50$, aggregated structures were produced and the same presented a large variation in zeta potential, being slightly positive on the average. These results suggest that the interaction between the structures led to disruption of liposomes, which by turn must have been incorporated in the structure of the bioparticles further leading to the formation of larger aggregates (Figure $\mathbf{3}$ ). This can be argued because the strong negative zeta potential of liposomes was no longer identified after ITC. Of notice, pKa of PG lipids was previously characterized being around 3.5 [39], which implies that in $\mathrm{pH} 2.50$ the lipid tends to neutralization. Despite, in the liposome bilayer the zeta potential was actually 
strongly negative suggesting that the close proximity of the lipids in the self-assembled membrane may provide a relative charge screening over the bilayer, thus maintaining the negative charge even under the pKa of the lipid. However, this must not be the case after membrane disruption and thereby, the vanishing of the negative zeta potential further confirms, along with formation of aggregates, the effective disruption of the liposomes in contact with the bioparticles. These results imply that the bioparticles present effective and strong interaction with negative lipid bilayers, even leading to the disruption of the later. In the specific $\mathrm{pH} 2.50$ the strong interaction must be due the chitosan whose protonated amine groups provide electrostatic interaction with the negatively charged lipids.

In pH 4.50 two populations of particles were produced before ITC and, and after interaction with liposomes, again two populations were found, but one with relative reduced average size (Table 1). For the same resulting structures, zeta potential was slightly negative because in $\mathrm{pH}$ 4.50 , even with disruption of liposomes, POPG must maintain the negative charges.

In pH 6.50 and 7.80 only one population of structures was found in both conditions and these structures were of reduced size if compared to the original bioparticles before ITC. Additionally, the resulting zeta potential after ITC was strongly negative. These results are intriguing and may suggest two scenarios (Figure 3). One could be the disruption of liposomes and incorporation of the lipids in the bioparticles structure; however, in the conditions of $\mathrm{pH}$ over the pKa of POPG it is apparent that the negative charge of the lipids play an important role in the structuration of the resulting particles. Indeed, as shown by TEM (Figure 2), the structure of the sole bioparticle consists in a random form with polymer branches hanging from the main structure and this configuration actually leads to the larger colloidal size distribution characterized by DLS. After mixing with liposomes, the final structure appeared relatively reduced denoting that the incorporation of the lipids must have enveloped the extended branches in the main central structure, hence reducing hydrodynamic diameters. The lipids apolar tails must be thus incorporated in the bioparticle by hydrophobic interactions with partially ( $\mathrm{pH} \mathrm{4.50)}$ or almost fully deprotonated ( $\mathrm{pH} 6.50$ and 7.80) segments of CHarg, since pKa of chitosan amine is around 6 [40]. With this configuration, the POPG polar heads must be placed on the surface of newly formed poly-lipid structure, which justifies the resulting negative zeta potential. The large variation of zeta potential must besides be related to the random distribution of lipids between bioparticles.

The second scenario, which is simpler, would be compaction and encapsulation of the bioparticles into the liposomes aqueous core (Figure 3 ). In this case, the interaction with the polysaccharides would partially disrupt the lipid membrane, but the lipid bilayer selfassembling in aqueous media would provide the restructuration of the membrane while compacting and "swallowing" the bioparticle into the new liposome core. Both scenarios may occur in higher or lower relative proportion depending on the $\mathrm{pH}$ of the dispersion, but indeed, the results of DLS and zeta potential confirm strong interaction between the bioparticles and the negative liposomes, evidenced by the significant size and surface charge alterations.

\subsubsection{Thermodynamic interaction}


In order to quantify the magnitude of this interaction, the heat released during titration was obtained by ITC. Figure $4 a$ and $\mathbf{b}$ show in the upper panels the heat released in the interactions of the bioparticle with the membrane model under controlled conditions of $\mathrm{pH}$ and temperature. The sharp descending peaks represent the heat released in each addition of the bioparticle suspension to the liposome suspension, showing a plain exothermic process. After a certain number of additions, the heat released decreased and disappeared, which indicates that there is no more interaction due to the saturation of membrane binding sites, leaving only the heat exchange by dilution, which is subtracted from the data analysis [8]. The bottom panels show the respective integrated heats and the solid sigmoid curves are the adjustments according to the model for a single set of binding sites [31]. In applying this model, one assumes the interaction between monomeric units of $\mathrm{CHarg}$ and the lipids influenced by the electrostatic forces between positive charges on CHarg and negative charges of POPG polar heads. Thereby, the number of binding sites $N$ represents the number of $\mathrm{CHarg}$ monomers "bound" to POPG molecules at saturation of binding sites. As shown in Table 2, this numbers are low for both $\mathrm{pH} 2.50$ and 4.50, however, when analyzing in terms of $1 / \mathrm{N}$, i.e., the number of POPG per CHarg monomers, one obtains 709 in pH 2.50 and 194 in pH 4.50. This huge amount of lipids per monomer is unrealistic, but it evidences the complex nature and the high intensity of the interaction of the entangled structure of the bioparticle with the lipid bilayer membrane.

This interpretation is further corroborated by the binding constant $K$, which shows high binding strengths between the bioparticle and the negatively charged lipid in acidic conditions (Table 2). The magnitude of $K$ suggests an irreversible process also corroborated with the significant decrease in Gibbs energy, which by turn was strongly dependent on enthalpy decrease rather than the entropic contribution.

For $\mathrm{pH} 6.50$ and 7.80 there was no saturation of the binding sites in the experimental conditions used in the ITC (Figure $\mathbf{4 c}$ and $\mathbf{d}$ ). Therefore, it was not possible to calculate all thermodynamic data. Even so, the interaction between the bioparticles and the liposomes was equally exothermic and regular for each titration, which allowed the estimation of the enthalpy variation (Table 2). It is noticed that there was a significant reduction in the variation of enthalpy comparing $\mathrm{pH} 6.50$ with 4.50 and a further reduction in $\mathrm{pH} 7.80$. These results also suggest a high thermodynamic interaction between the bioparticle and liposome in $\mathrm{pH}$ conditions close to neutrality and alkaline, which must have contributed to the liposome disruption and compaction and incorporation of the bioparticle in the liposome (Figure 3), as pointed out by the DLS results that determined a significant reduction in colloidal sizes and the highly negative zeta potential, characteristic of the POPG membrane.

It is also worth mentioning the importance of arginine bonded on chitosan (Figure 1), since arginine guanidinium group has a high cationic character that must interact with the negative charge of POPG. In fact, a pKa of around 13.8 has been described for arginine [29], which means that in all $\mathrm{pH}$ conditions employed in this study, the strong cationic character of arginine prevails. Therefore, arginine provides a permanent electrostatic characteristic to chitosan, which must have influenced the interaction with the membrane model. 
In general, the results show that the interaction of the bioparticle with the membrane model is thermodynamically favored in the studied $\mathrm{pHs}$ even under conditions of low ionic concentration $(8 \mathrm{mM})$, also suggesting that the material may present a high interaction with cell membranes, which can promote cell internalization.

\subsection{Determination of praziquantel entrapment}

As a standard procedure, the drug entrapment was evaluated by spectrophotometric analysis. In order to determine the percentage of drug incorporation in the bioparticles, a standard curve was constructed (Figure 5) with the results of absorbance of praziquantel solutions in methanol:water (60:40) comparing with the absorbance of the solution of the bioparticle, that is, the bioparticle-free supernatant obtained by centrifugation and with the adequate proportion of methanol. Figure $\mathbf{5}$ shows that the solution in which the lyophilized bioparticles containing the drug were dispersed had an absorbance (square icon) almost equivalent to the absorbance of the solution containing $5 \%$ of the concentration of praziquantel included in the preparation of the bioparticles. Therefore, by deduction, the results of the experiment show that $95 \%$ of the drug was incorporated in the structure of the bioparticle. This result means a high content of drug encapsulation in the carrier, which shows its potential for technological development and application.

\subsection{In vivo application}

\subsubsection{Animal model and parasite infection}

The Corydoras schwartzi (Figure 6a) is a catfish belonging to the Callichthyidae family. It was chosen as in vivo animal model to study the application of the bioparticles as effective oral drug delivery devices due the fact that this fish species was naturally found with high levels of intestinal parasites infection. The sampled fish presented an average of $2.37 \mathrm{~g}$ of body weight, $4.5 \mathrm{~cm}$ in length and an average of 34.1 intestinal parasites per fish, as determined from the control group. The parasite was a digenetic trematode, which constitutes a major helminth group that parasitize humans and animals. It was found in the whole intestinal tract as free metacercariae in adult stage (Figure $\mathbf{6 b}$ and $\mathbf{c}$ ), and as encysted metacercariae (Figure $\mathbf{6 d}$ ). The cyst is a subspherical capsule containing fluids, adult and the early stages of the parasite development, as shown in Figure $6 \mathbf{d}$, left side structure. Typically, the cysts presented diameters varying between $400 \mu \mathrm{m}$ and $2 \mathrm{~mm}$ while adult trematode presented between 300 $\mu \mathrm{m}$ and $1.5 \mathrm{~mm}$ in length.

\subsubsection{Oral administration of bioparticles}

The bioparticles oral administration to Corydoras fish was efficiently performed thanks to the high compliance to the produced material (see video at [28]). Indeed, the bioparticle presents adequate texture and palatability to diverse fish of different ecologic niches as we previously reported [28]. There was also no mortality and no behavior changes of the fish during the entire experiment. Besides, no clinical abnormalities were observed in the fish provided orally with the bioparticles and with praziquantel-bioparticles, evidencing high tolerability. 
After administration, the examination of fish intestines revealed well spread particles along the whole intestinal tract and besides on the intestinal parasites, as shown in Figure 7. For the fish examined after $1 \mathrm{~h}$ of praziquantel-bioparticles administration (Figure 7a), the digenetic trematodes appeared alive with apparently normal movements, no physical alterations and with dim fluorescent spots in their internal organs. The same was observed for the bioparticles free of drug. However, after $6 \mathrm{~h}$ (Figure 7b) bioparticles were found in highly concentrated spots on the intestinal walls, but most importantly, they were found well inside the parasites body, spread in internal organs. Furthermore, it was noticeable that for praziquantelbioparticles some parasites presented alterations is movements and in physical aspect, specially for those with apparent higher internal concentration of bioparticles, as can be seen for the parasite on the right side of Figure $\mathbf{7 b}$. After 24 of administration, the parasites showed even more internalized bioparticles and apparent degraded physical aspect (see the three parasites in the upper right side of Figure 7c) and short or null movements. Figure 7d shows an amplified section of metacercariae body evidencing internal organs highly filled with bioparticles. Of notice, the parasites physical alterations were observed only for praziquantelbioparticles, evidencing the effect of the anthelmintic drug.

Furthermore, in the same periods, the presence of bioparticles is also observed in the resistant form of these parasites, that is, in the cysts (Figure 8). As evidenced in Figure 8a, the bioparticles cover the cyst membrane on both external and internal surface, are dispersed in the cyst internal fluids, and importantly, are found in internal organs of the metacercariae grooving inside the cyst (see the two well focused parasites). Figure $\mathbf{8 b}$ shows another cyst containing early stage metacercariae and also with internalized bioparticles. This cyst is burst in on its left side, which may have possibly resulted from the anthelmintic action of praziquantel and/or disruption influenced by the bioparticles.

These results all together evidence an effective membrane-penetrating process of the bioparticles, which by turn may allow an efficient treatment of the parasite infection by the drug release directly on the digenetic trematodes, providing its total elimination from the host organism. The membrane-penetrating characteristic must be related to the mucoadhesive properties of $\mathrm{CHarg}$ and $\mathrm{ALG}$, which even together in the same particle maintain this propitious quality. Indeed, the presence of arginine moieties on chitosan (Figure 1), with permanently positive charged guanidinium groups, must have improved the membranepenetrating features, since arginine is well known as a cell-penetrating molecule. Additionally, ALG with negatively charged carboxyl groups in intestinal $\mathrm{pH}$, must besides contribute to the membrane-penetrating process with electrostatic interaction and repulsion with the complex nature of proteins composing living membranes. It is well known that sialic acid from glycoconjugates, phosphate groups from phospholipids, carboxyl groups of amino acids and sulfated polysaccharides associated with the plasma membrane contribute to the cell surface charge of living organisms [41,42]. Nevertheless, taking in account the strong thermodynamic interaction of the bioparticles with the model of negatively charged lipid membrane and the effective in vivo membrane-penetrating characteristic, it is evidenced that the bioparticles 
interaction with biological membranes is far more complex than simple charge-charge interactivities.

\subsubsection{Intestinal parasites survey and treatment}

In order to evaluate the practical performance of the bioparticles for drug delivery, we conducted the in vivo experiments in which the lyophilized bioparticles were systematically applied via oral administration to Corydoras schwartzi fish (Figure 6).

Indeed, all Corydoras were highly infected with metacercariae of digenetic trematodes, showing a prevalence $P=100 \%$. As shown in Figure 6 , metacercariae were found in cysts and as free circulating metacercariae throughout the whole intestinal tract.

Nevertheless, the fish submitted to treatment with the anthelmintic praziquantel transported in the bioparticles showed a persistent reduction in parasite indices during the first weeks after the oral administration of the drug carriers. In terms of prevalence, the same was $P=$ $10 \%$ at the third week, i.e., $90 \%$ of the treated fish were found completely clean of parasites. Moreover, as evidenced in Figure 9, the mean intensity (MI) showed a significant average reduction from 34.1 to 18.2 parasites at 7 days from the beginning of administration and to 2.1 parasites at 14 days of administration, keeping approximately this lower number after the following three weeks. This represents a reduction of over $97 \%$ of MI. The initial high standard deviation was equally expressively reduced from the $14^{\text {th }}$ day on.

It was further observed that at day 7 the majority of parasites were found apparently dead or in advanced stage of decomposition, as shown for the metacercariae in the cysts shown in Figure 10.

These excellent results evidence an effective treatment of the parasite infection highlighting the efficiency of the drug delivery bioparticles, which apparently carried the drug and delivered the same in the targeted side of its expected action. Concerning the pharmacological action, the anthelmintic praziquantel is known to act mainly through calcium channels, increasing the permeability of the parasite membranes and allowing the entry of $\mathrm{Ca}^{+2}$ [43]. This mechanism is believed to occur due to the interaction of the drug with lipid molecules in the parasitic integumentary membrane. The increase in calcium ends up increasing the muscle contractions of the parasites and consequently it causes spastic muscle paralysis and disintegration of the integument [44], as can be seen in Figure 10. In this way, the helminths detach from the intestine and are metabolized and eliminated from the host's body [43-45]. The efficient treatment unveiled in the study points out that the concentration of praziquantel, $24.2 \mathrm{mg} / \mathrm{kg}$ b.v., was sufficient to reach the prominent results considering that the bioparticles provided the transport and delivery of the drug in the expected site of action and thereby promoted the effective treatment.

As a matter of fact, the bioparticles have been shown to reaching and being able to adhere to the intestinal tract, thanks to the mucoadhesion [28], are able to penetrate the membranes of cyst and metacercariae of digenetic trematodes and capable of transporting and releasing a drug at the target site. This study evidences the high potential of the CHarg/ALG bioparticles as an applicable oral drug delivery material. 


\section{Conclusion}

The bioparticle interaction with the liposome of negatively charged lipid membrane led to the membrane disruption providing the formation of aggregates in conditions of high and moderate acidity, however the formation of highly negative surface charge nano-aggregates under conditions of low acidity and alkaline, with membrane disruption, compacting of the bioparticle and internalization in the liposome core. In the thermodynamic process, a strong and energetically favored interaction was evidenced in all $\mathrm{pH}$ conditions. The high potential for drug incorporation in the bioparticle was also demonstrated with a 95\% entrapment of praziquantel. The in vivo study using Corydoras fish as an animal model showed the applicability of the bioparticle as a drug carrier, with easy administration of the lyophilized powder, high acceptance by the oral route and effective treatment of intestinal parasite infection. Hence, the bioparticle was highly efficient in the transport and release of praziquantel at the target site, promoting effective action of the anthelmintic.

These studies evidence the potential of the bioparticle in penetrating biological membrane and providing the transport and in vivo release of bioactive. Therefore, it consists of a biomaterial of effective application in drug delivery.

\section{Author contributions}

P.D. Mathews: Conceptualization, Experiment, Data acquisition, Formal analysis, Supervision, Project administration, Writing-original draft. A.C.M.F. Patta: Experiment, Data acquisition, Formal analysis, Writing-original draft. R.R.M. Madrid: Experiment, Data acquisition, Formal analysis. C.A.B. Ramirez: Experiment, Data acquisition, Formal analysis. O. Mertins: Conceptualization, Formal analysis, Writing-original draft, Supervision, Project administration, Writing-review \& editing, Funding acquisition.

\section{Declaration of competing interest}

The authors declare no competing interests.

\section{Acknowledgements}

The authors thank Prof. M.A. Juliano for providing lyophilization equipment, Prof. K.A. Riske for ITC and DLS equipment, and Dr. T.S. Backer for revising the grammar of the text. The Sao Paulo Research Foundation (FAPESP, Brazil) is acknowledged for MSc. scholarship to A. C.M.F.P. (grant: 2017/16722-6), Post-doc fellowship to P.D.M. (grant: 2018/20482-3) and research grant and financial support to O.M. (grants: 2015/23948-5 and 2016/13368-4). R.R.M.M. and C.A.B.R. thank CAPES, Brazil, for a MSc. scholarship.

\section{Data availability statement}

Data will be available on request. 


\section{References}

[1] Souhaila H. El Moukhtari, Carlos Rodríguez-Nogales, María J. Blanco-Prieto. Oral lipid nanomedicines: current status and future perspectives in cancer treatment. Advanced Drug Delivery Reviews (2021) https://doi.org/10.1016/j.addr.2021.03.004

[2] Tyler D. Brown, Kathryn A. Whitehead, Samir Mitragotri. Materials for oral delivery of proteins and peptides. Nature Reviews Materials 5, 127-148 (2020).

[3] Xiangfei Han, Yang Lu, Jinbing Xie, Ershuai Zhang, Hui Zhu, Hong Du, Ke Wang, Boyi Song, Chengbiao Yang, Yuanjie Shi, Zhiqiang Cao. Zwitterionic micelles efficiently deliver oral insulin without opening tight junctions. Nature Nanotechnology 15, 605-614 (2020).

[4] Susan Hua BPharm, Ellen Marks, Jennifer J. Schneider BPharm, Simon Keely. Advances in oral nano-delivery systems for colon targeted drug delivery in inflammatory bowel disease: Selective targeting to diseased versus healthy tissue. Nanomedicine: Nanotechnology, Biology and Medicine 11, 1117-1132 (2015).

[5] Suraj Sharma, Gunjan Sarkar, Bhupendra Srestha, Dipankar Chattopadhyay, Manas Bhowmik. In-situ fast gelling formulation for oral sustained drug delivery of paracetamol to dysphagic patients. International Journal of Biological Macromolecules 134, 864-868 (2019).

[6] Nurfazreen Anuar, Akmal H. Sabri, Tommy Julianto Bustami Effendi, Khuriah Abdul Hamid. Development and characterisation of ibuprofen-loaded nanoemulsion with enhanced oral bioavailability. Heliyon 6, e04570 (2020).

[7] Kim, K. S., Suzuki, K., Cho, H., Youn, Y. S., Bae, Y. H. Oral nanoparticles exhibit specific highefficiency intestinal uptake and lymphatic transport. ACS Nano. 12, 8893-8900 (2018).

[8] Patrick D. Mathews, Ana C.M. Fernandes Patta, Joao V. Goncalves, Gabriella dos Santos Gama, Irene Teresinha Santos Garcia, Omar Mertins. Targeted drug delivery and treatment of endoparasites with biocompatible particles of $\mathrm{pH}$-responsive structure. Biomacromolecules 19, 499-510 (2018).

[9] Carmen Gómez-Guillén, Pilar Montero. Enhancement of oral bioavailability of natural compounds and probiotics by mucoadhesive tailored biopolymer-based nanoparticles: a review. Food Hydrocolloids, 106772 (2021).

[10] Jonas Reinholz, Katharina Landfester, Volker Mailänder. The challenges of oral drug delivery via nanocarriers. Drug Delivery 25, 1694-1705 (2018). 
[11] Yu-Sang Sabrina Yang, Kelly D. Moynihan, ..., Darrell J. Irvine. Targeting small molecule drugs to $T$ cells with antibody-directed cell-penetrating gold nanoparticles. Biomaterials Science 7, 113-124 (2019).

[12] Sungsook Ahn, Eunseok Seo, Kihean Kim, Sang Joon Lee. Controlled cellular uptake and drug efficacy of nanotherapeutics. Scientific Reports 3, 1997 (2013).

[13] Eunji Jo, June Seok Heo, Ja-Yun Lim, Bo-Ram Lee, Chul Joo Yoon, Jinkwan Kim, Jeewon Lee. Peptide ligand-mediated endocytosis of nanoparticles to cancer cells: Cell receptorbinding- versus cell membrane-penetrating peptides. Biotechnology and Bioengineering 115, 1437-1449 (2018).

[14] Prasanta Panja, Nikhil R. Jana. Arginine-terminated nanoparticles of $<10 \mathrm{~nm}$ size for direct membrane penetration and protein delivery for straight access to cytosol and nucleus. J. Phys. Chem. Lett. 11, 2363-2368 (2020).

[15] Nasir Hussain, Vikas Jaitley, Alexander T. Florence. Recent advances in the understanding of uptake of microparticulates across the gastrointestinal lymphatics. Advanced Drug Delivery Reviews 50, 107-42 (2001).

[16] Laurence Plapied, Nicolas Duhem, Anne des Rieux, Véronique Préat. Fate of polymeric nanocarriers for oral drug delivery. Current Opinion in Colloid and Interface Science 16, 228237 (2011).

[17] Marlene Almeida Lopes, Bárbara Azevedo Abrahim, ..., António José Ribeiro. Intestinal absorption of insulin nanoparticles: Contribution of M cells. Nanomedicine: Nanotechnology, Biology and Medicine 10, 1139-1151 (2014).

[18] Anne des Rieux, Virginie Fievez, Marie Garinot, Yves-Jacques Schneider, Véronique Préat. Nanoparticles as potential oral delivery systems of proteins and vaccines: a mechanistic approach. Journal of Controlled Release 116, 1-27 (2006).

[19] Shu-jun Cao, Shuo Xu, ..., Xiang-hong Sun. Nanoparticles: Oral delivery for protein and peptide drugs. AAPS Pharm Sci Tech 20, 190 (2019).

[20] John D. Smart. The basics and underlying mechanisms of mucoadhesion. Advanced Drug Delivery Reviews 57, 1556-1568 (2005).

[21] Ioannis A. Sogias, Adrian C. Williams, Vitaliy V. Khutoryanskiy. Why is chitosan mucoadhesive? Biomacromolecules 9, 1837-1842 (2008). 
[22] Patrick D. Mathews, Omar Mertins. Chitosan and lipid composites as versatile biomedical material. In: A.M. Holban, A.M. Grumezescu (eds). Materials for Biomedical Engineering: Nanomaterials-Based Drug Delivery. 259-291 (2019) Elsevier. https://doi.org/10.1016/B9780-12-816913-1.00009-X

[23] Omar Mertins, Rumiana Dimova. Insights on the interactions of chitosan with phospholipid vesicles. Part II: membrane stiffening and pore formation. Langmuir 29, 14552-14559 (2013).

[24] Bianca B.M. Garcia, Omar Mertins, Emerson R. da Silva, Patrick D. Mathews, Sang W. Han. Arginine-modified chitosan complexed with liposome systems for plasmid DNA delivery. Colloids and Surfaces B: Biointerfaces 193, 111131 (2020).

[25] Sanjib Sarkar, Dibyendu Das, Prachurjya Dutta, Jatin Kalita, Sawlang Borsingh Wann, Prasenjit Manna. Chitosan: A promising therapeutic agent and effective drug delivery system in managing diabetes mellitus. Carbohydrate Polymers 247, 116594 (2020).

[26] Tzyy-Harn Yeh, Li-Wen Hsu, Michael T. Tseng, Pei-Ling Lee, Kiran Sonjae, Yi-Cheng Ho, Hsing-Wen Sung. Mechanism and consequence of chitosan-mediated reversible epithelial tight junction opening. Biomaterials 32, 6164-6173 (2011).

[27] Kesinee Netsomboon, Andreas Bernkop-Schnürch. Mucoadhesive vs. mucopenetrating particulate drug delivery. European Journal of Pharmaceutics and Biopharmaceutics 98, 7689 (2016).

[28] Ana C.M. Fernandes Patta, Patrick D. Mathews, Rafael R.M. Madrid, Vera L.S. Rigoni, Emerson R. Silva, Omar Mertins. Polyionic complexes of chitosan- $\mathrm{N}$-arginine with alginate as $\mathrm{pH}$ responsive and mucoadhesive particles for oral drug delivery applications. International Journal of Biological Macromolecules 148, 550-564 (2020).

[29] C.A. Fitch, G. Platzer, M. Okon, B.E. Garcia-Moreno, L.P. Mclntosh. Arginine: its pKa value revisited. Protein Science 24, 752-761 (2015).

[30] Rafael R.M. Madrid, Patrick D. Mathews, Ana C.M.F. Patta, Anai P. Gonzales-Flores, Carlos A.B. Ramirez, Vera L.S. Rigoni, Marcos Tavares-Dias, Omar Mertins. Safety of oral administration of high doses of ivermectin by means of biocompatible polyelectrolytes formulation. Heliyon 7, e05820 (2021).

[31] Omar Mertins, Rumiana Dimova. Binding of chitosan to phospholipid vesicles studied with isothermal titration calorimetry. Langmuir 27, 5506-5515 (2011). 
[32] B.J. Berne, R. Pecora. Dynamic Light Scattering: With Applications to Chemistry, Biology, and Physics. Dover Publications, New York, 2000.

[33] Ruben C. Cuadros, Norma L.S. Rivadeneyra, Anai Flores-Gonzales, Omar Mertins, Jose C.O. Malta, M. Enrique Serrano-Martínez, Patrick D. Mathews. Intestinal histological alterations in farmed red-bellied pacu Piaractus brachypomus (Characiformes: Serrasalmidae) heavily infected by roundworms. Aquaculture International (2021) https://doi.org/10.1007/s10499021-00670-0

[34] Albert O. Bush, Kevin D. Lafferty, Jeffrey M. Lotz, Allen W. Shostak. Parasitology meets ecology on its own terms: Margolis et al. revisited. Journal of Parasitology 83, 575-583 (1997).

[35] Andreas F. Thünemann, Martin Müller, Herbert Dautzenberg, Jean-François Joanny, Hartmut Löwen. Polyelectrolyte Complexes. In: Manfred Schmidt (ed) Polyelectrolytes with Defined Molecular Architecture II. Advances in Polymer Science 166, 113-171 (2004) Springer, Berlin, Heidelberg. https://doi.org/10.1007/b11350

[36] Javier P. Quiñones, Hazel Peniche, Carlos Peniche. Chitosan based self-assembled nanoparticles in drug delivery. Polymers 10, 235 (2018).

[37] Simona Morariu, Cristina-Eliza Brunchi, Maria Bercea. The behavior of chitosan in solvents with different ionic strengths. Industrial and Engineering Chemistry Research 5, 12959-12966 (2012).

[38] Lan Ding, Yan Huang, XiXi Cai, Shaoyun Wang. Impact of $\mathrm{pH}$, ionic strength and chitosan charge density on chitosan/casein complexation and phase behavior. Carbohydrate Polymers 208, 133-141 (2019).

[39] Nabil A. Alhakamy, Ibrahim Elandaloussi, Saba Ghazvini, Cory J. Berkland, Prajnaparamita Dhar. Effect of lipid headgroup charge and $\mathrm{pH}$ on the stability and membrane insertion potential of calcium condensed gene complexes. Langmuir 31, 4232-4245 (2015).

[40] Q.Z. Wang, X.G. Chen, N. Liu, S.X. Wang, C.S. Liu, X.H. Meng, C.G. Liu. Protonation constants of chitosan with different molecular weight and degree of deacetylation. Carbohydrate Polymers 65, 194-201 (2006).

[41] Rossiane C. Vommaro, Marcia Attias, Fernando C. Silva Filho, Patrick T.K. Woo, Wanderley de Souza. Surface charge and surface carbohydrates of cryptobia salmositica virulent and avirulent forms and of C. bullocki (Kinetoplastida: Cryptobiidae). Parasitology Research 83, 698-705 (1997). 
[42] Richard W. Burry, John G. Wood. Contributions of lipids and proteins to the surface charge of membranes. Journal of Cell Biology 82, 726-741 (1972).

[43] R.J. Martin. Modes of action of anthelmintic drugs. The Veterinary Journal 154, 11-34 (1997).

[44] H. Björklund, G. Bylund. Absorption, distribution and excretion of the anthelmintic praziquantel (Droncit) in rainbow trout (Salmo gairdneri R.). Parasitology Research 73, 240244 (1987).

[45] E. Zuskova, V. Piackova, J. Machova, L. Chupani, C. Steinbach, A. Stara, J. Velisek. Efficacy and toxicity of praziquantel in helminth-infected barbel (Barbus barbus L.). Journal of Fish Diseases 41, 643-649 (2018).

\section{Captions}

Table 1. DLS and zeta potential results as function of $\mathrm{pH}$ for particles composed of biopolymers chitosan- $N$-arginine and alginate (CHarg/ALG), POPG liposomes and the produced structures after interaction of both in the ITC chamber. Variations are standard deviations as obtained for each analysis.

Table 2. Thermodynamic parameters characterizing the interaction between CHarg/ALG particles and POPG liposomes at $25^{\circ} \mathrm{C}$ in different $\mathrm{pH}$ conditions.

Figure 1. Molecular structure of praziquantel; segment of protonated chitosan- $N$-arginine with highlighted guanidinium group; and ionized alginate.

Figure 2. Transmission electron microscopy of bioparticles in aqueous solution: (a) general aspect of random coiled particles of chitosan- $N$-arginine and alginate; (b) amplification of a typical bioparticle; (c) structure of aggregated bioparticles dispersed in $8 \mathrm{mM}$ buffer solution of $\mathrm{pH} 4.50$; (d) amplification of the aggregate with blue rings outlining two of the bioparticles which aggregated.

Figure 3. Scheme of possible scenarios of the structures obtained from the interaction of CHarg/ALG particles of slightly positive surface charge with POPG liposomes of negative surface charge: formation of larger aggregates and relative smaller poly-lipid structure and compacted bioparticle encapsulated in the liposome core. Structures are not in the same scale. See text for more details. 
Figure 4. ITC results for the interaction between CHarg/ALG particles with POPG liposomes at $25^{\circ} \mathrm{C}$ in $\mathrm{pH} 2.50$ (a), 4.50 (b), 6.50 (c) and 7.80 (d).

Figure 5. Variation of absorbance as a function of concentration of praziquantel in methanol:water $(60: 40, \mathrm{v}: \mathrm{v})$ solutions. The square is the absorbance corresponding to the solution separated from the bioparticles containing $38 \mu \mathrm{M}$ praziquantel. The solid line is guide to the eye.

Figure 6. Snapshot of Corydoras schwartzi fish (a). Photomicrographs of digenetic trematode in the fish intestine (b) and collected (c) and an intestinal trematode cyst containing metacercariae in yang and adult developmental stages (d). Scale bar expands $500 \mu \mathrm{m}$.

Figure 7. Confocal laser scanning micrographs (phase contrast, merge and epifluorescence of fluorescein isothiocyanate) of digenetic trematodes in intestines of Corydoras schwartzi after $1 \mathrm{~h} \mathrm{(a),} 6 \mathrm{~h} \mathrm{(b)}$ and $24 \mathrm{~h} \mathrm{(c)} \mathrm{of} \mathrm{oral} \mathrm{administration} \mathrm{of} \mathrm{fluorescently} \mathrm{labeled} \mathrm{chitosan-} N$-arginine and alginate bioparticles containing $38 \mu \mathrm{M}$ praziquantel. The amplification (d) evidences the presence of bioparticles in internal organs of a parasite from the picture above. Scale bar expands $500 \mu \mathrm{m}$.

Figure 8. Confocal laser scanning micrographs (phase contrast, merge and epifluorescence of fluorescein isothiocyanate) of cysts of digenetic trematodes in intestines of Corydoras schwartzi after $6 \mathrm{~h}$ of oral administration of fluorescently labeled chitosan- $N$-arginine and alginate bioparticles containing $38 \mu \mathrm{M}$ praziquantel. At least two metacercariae are focused inside the cyst in (a) while a cyst containing early stage metacercariae is burst on its left side in (b). Scale bar expands $500 \mu \mathrm{m}$ for all.

Figure 9. Mean intensity (MI) of intestinal parasites found in Corydoras fish during 35 days after the treatment with oral administration of praziquantel-bioparticles.

Figure 10. Digenetic trematode cysts found in intestine of Corydoras schwartzi containing parasites in advanced state of decomposition after one week of praziquantel-bioparticles administration. Scale bars expand $500 \mu \mathrm{m}$. 
Figures

\section{Figure 1.}<smiles>O=C(C1CCCCC1)N1CC(=O)N2CCc3ccccc3C2C1</smiles>

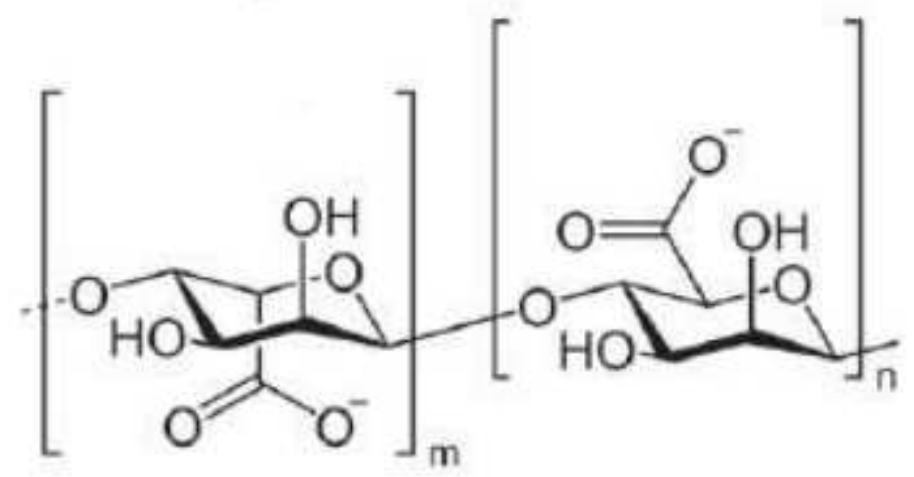

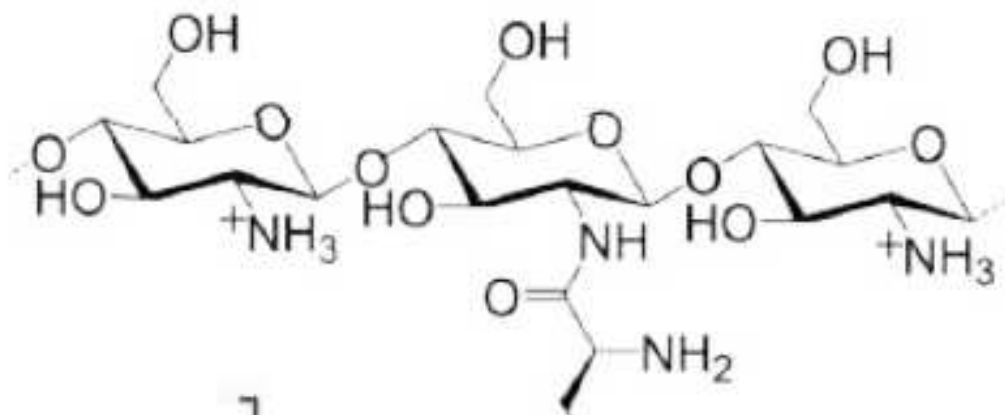

\section{Figure 1}

Molecular structure of praziquantel; segment of protonated chitosan-N-arginine with highlighted guanidinium group; and ionized alginate. 


\section{Figure 2.}
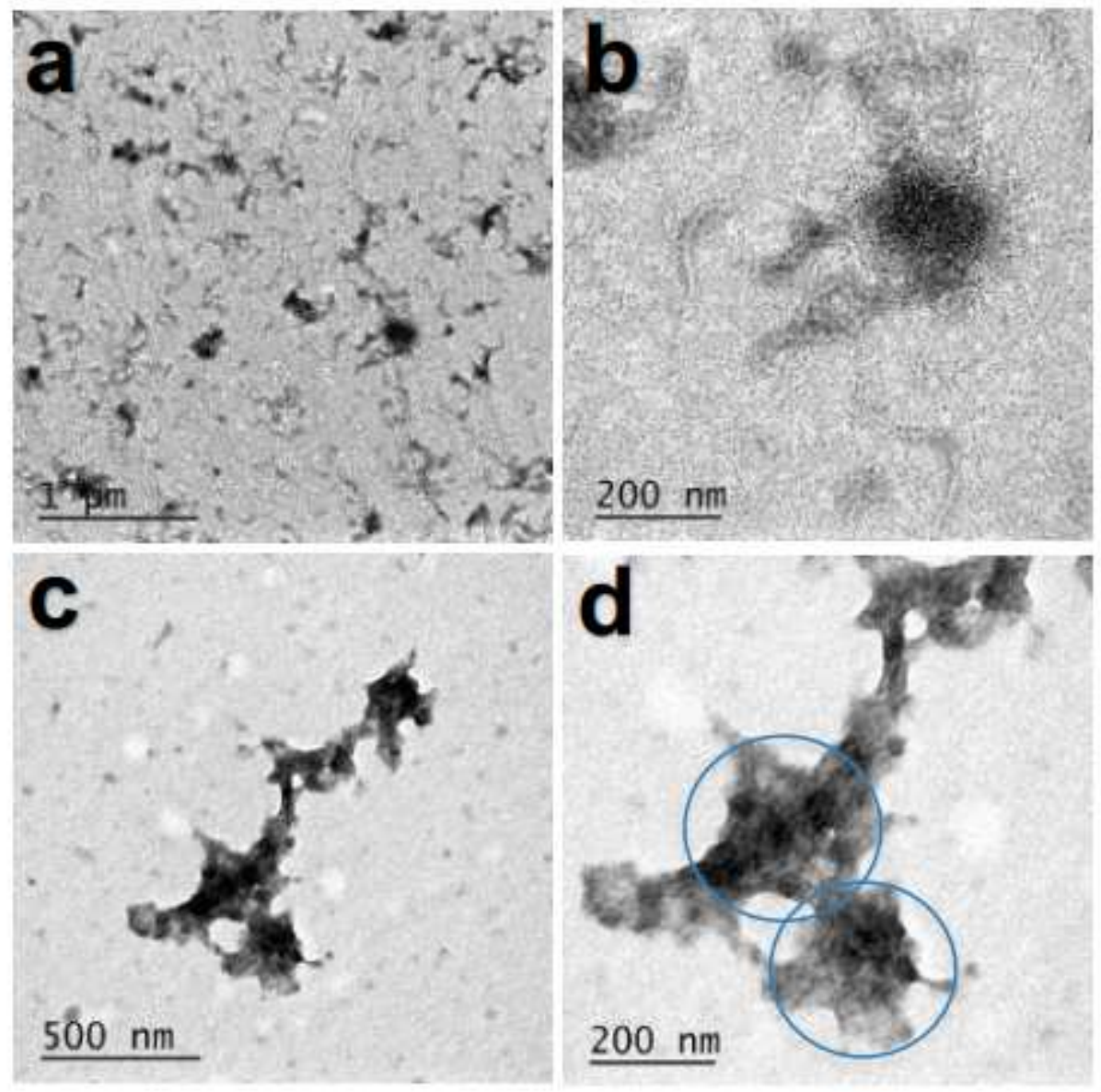

Figure 2

Transmission electron microscopy of bioparticles in aqueous solution: (a) general aspect of random coiled particles of chitosan-N-arginine and alginate; (b) amplification of a typical bioparticle; (c) structure of aggregated bioparticles dispersed in $8 \mathrm{mM}$ buffer solution of $\mathrm{pH} 4.50$; (d) amplification of the aggregate with blue rings outlining two of the bioparticles which aggregated. 


\section{Figure 3.}

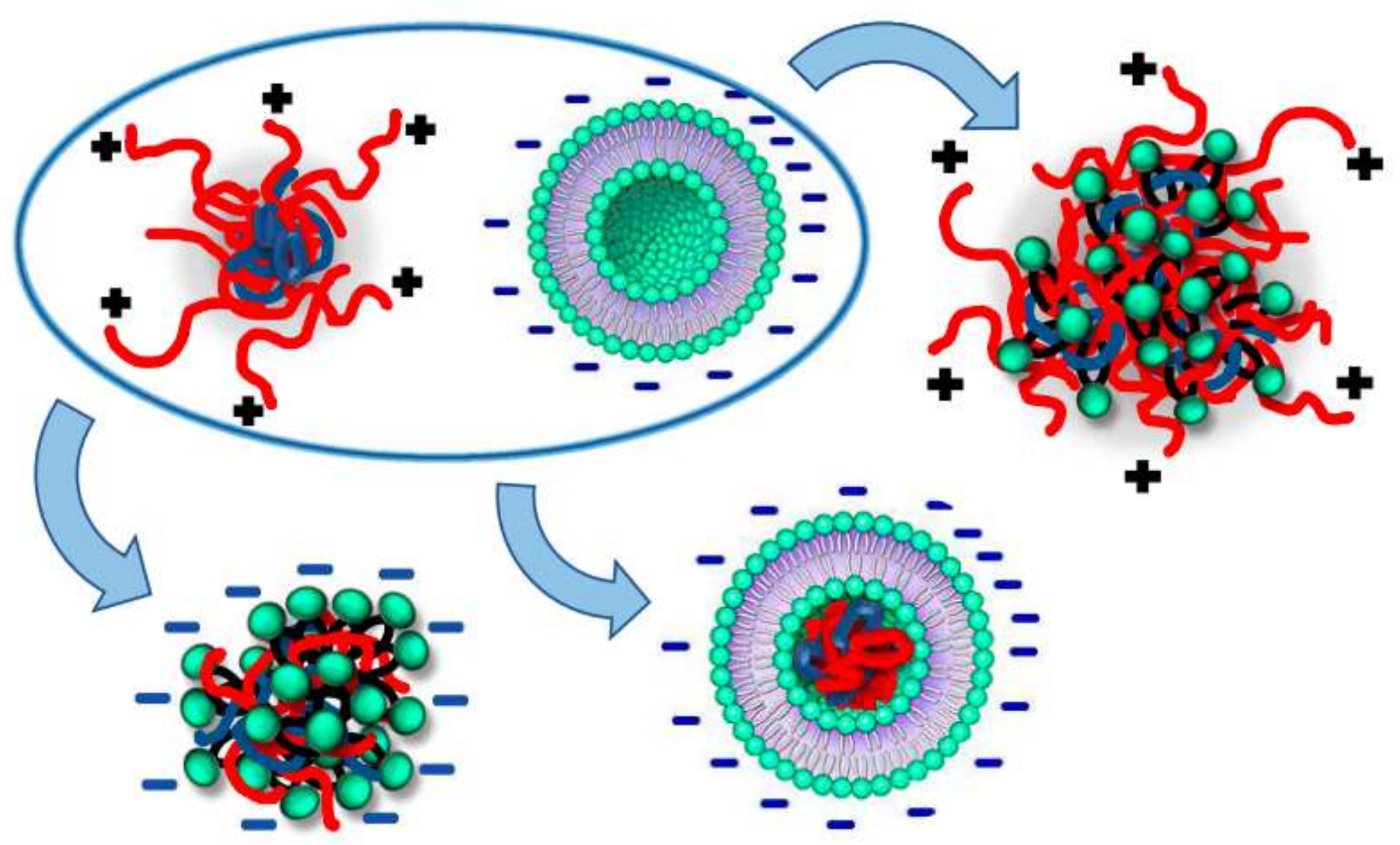

Figure 3

Scheme of possible scenarios of the structures obtained from the interaction of CHarg/ALG particles of slightly positive surface charge with POPG liposomes of negative surface charge: formation of larger aggregates and relative smaller poly-lipid structure and compacted bioparticle encapsulated in the liposome core. Structures are not in the same scale. See text for more details 
Figure 4.
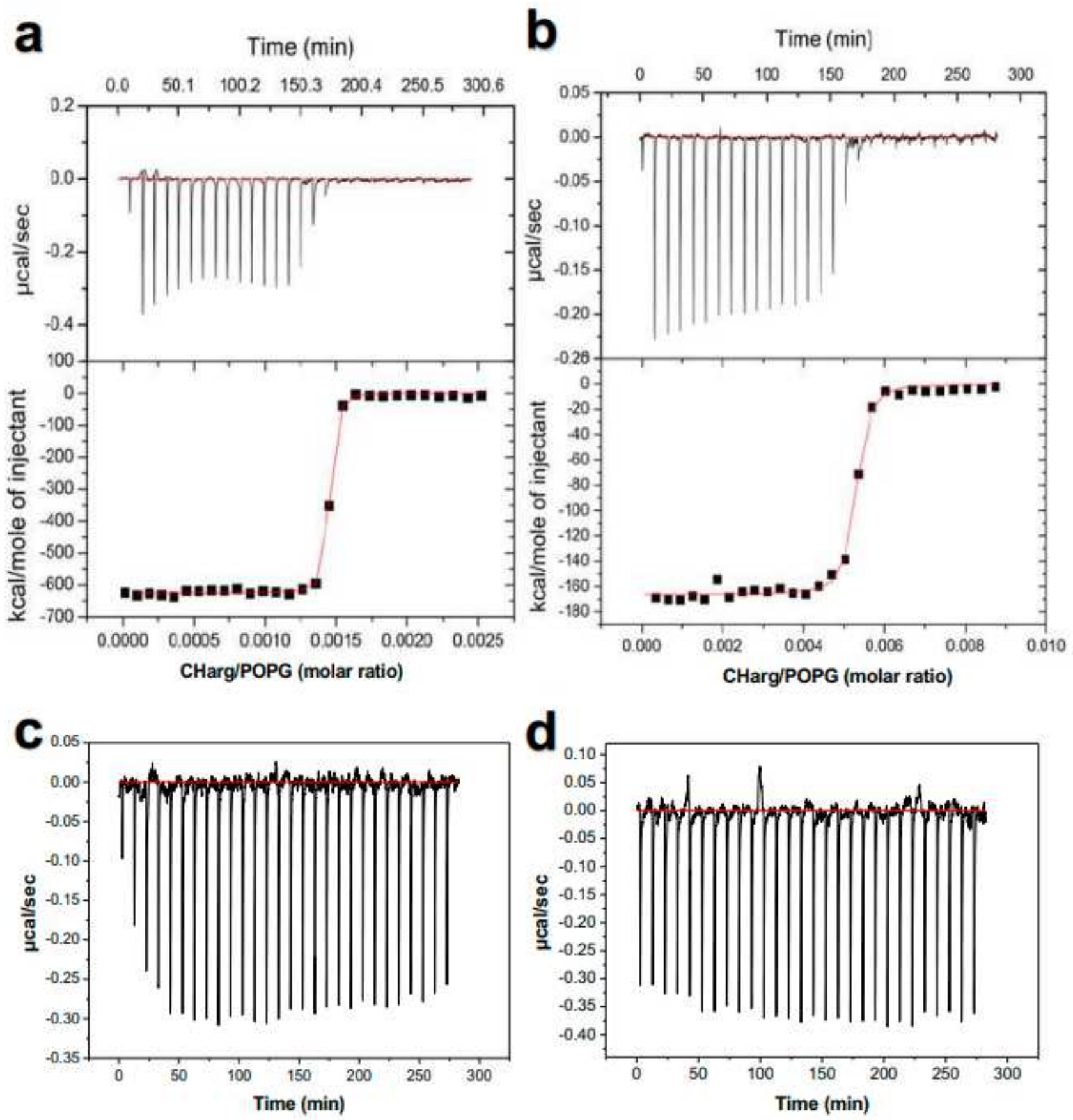

Figure 4

ITC results for the interaction between CHarg/ALG particles with POPG liposomes at $25^{\circ} \mathrm{C}$ in $\mathrm{pH} 2.50$ (a), 4.50 (b), 6.50 (c) and 7.80 (d). 
Figure 5.

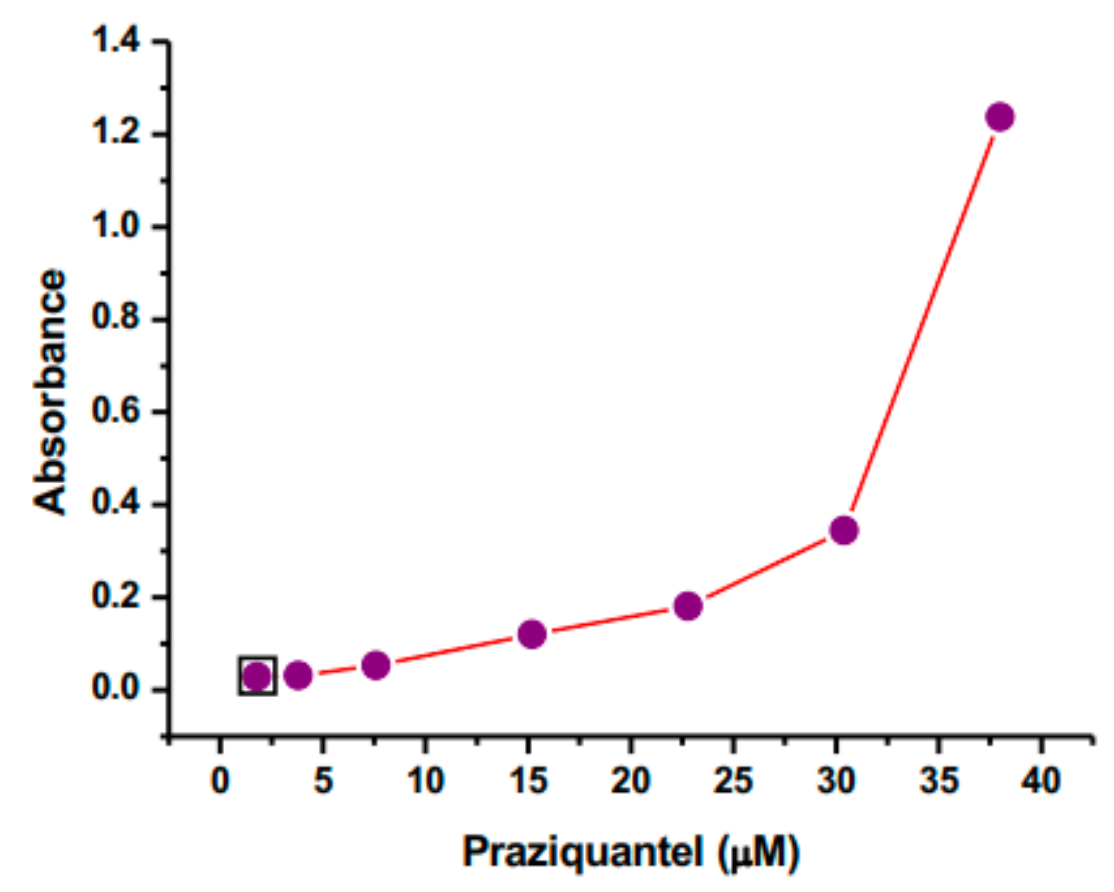

Figure 5

Variation of absorbance as a function of concentration of praziquantel in methanol:water (60:40, v:v) solutions. The square is the absorbance corresponding to the solution separated from the bioparticles containing $38 \mu \mathrm{M}$ praziquantel. The solid line is guide to the eye. 


\section{Figure 6.}

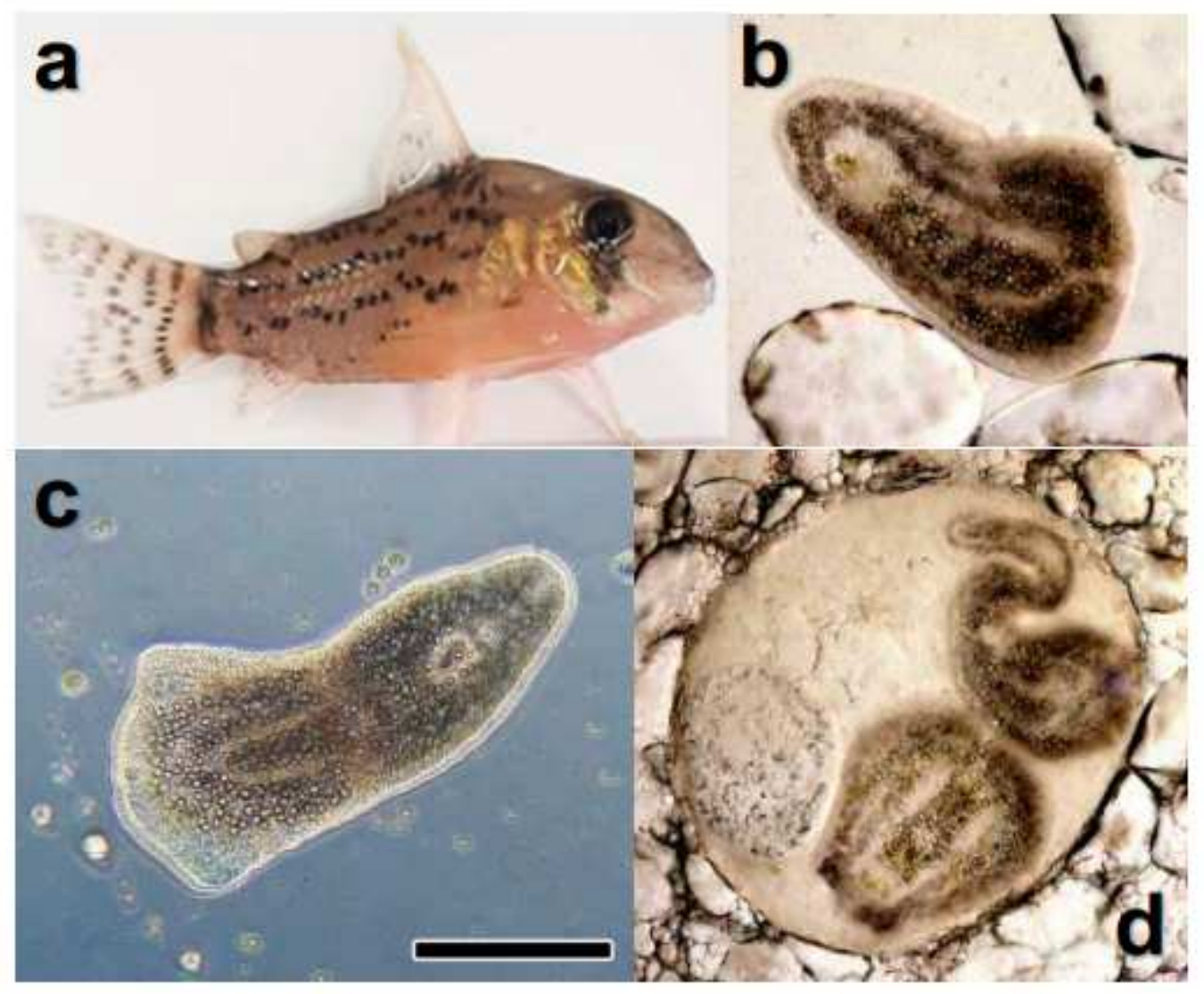

Figure 6

Snapshot of Corydoras schwartzi fish (a). Photomicrographs of digenetic trematode in the fish intestine (b) and collected (c) and an intestinal trematode cyst containing metacercariae in yang and adult developmental stages (d). Scale bar expands $500 \mu \mathrm{m}$. 
Figure 7.

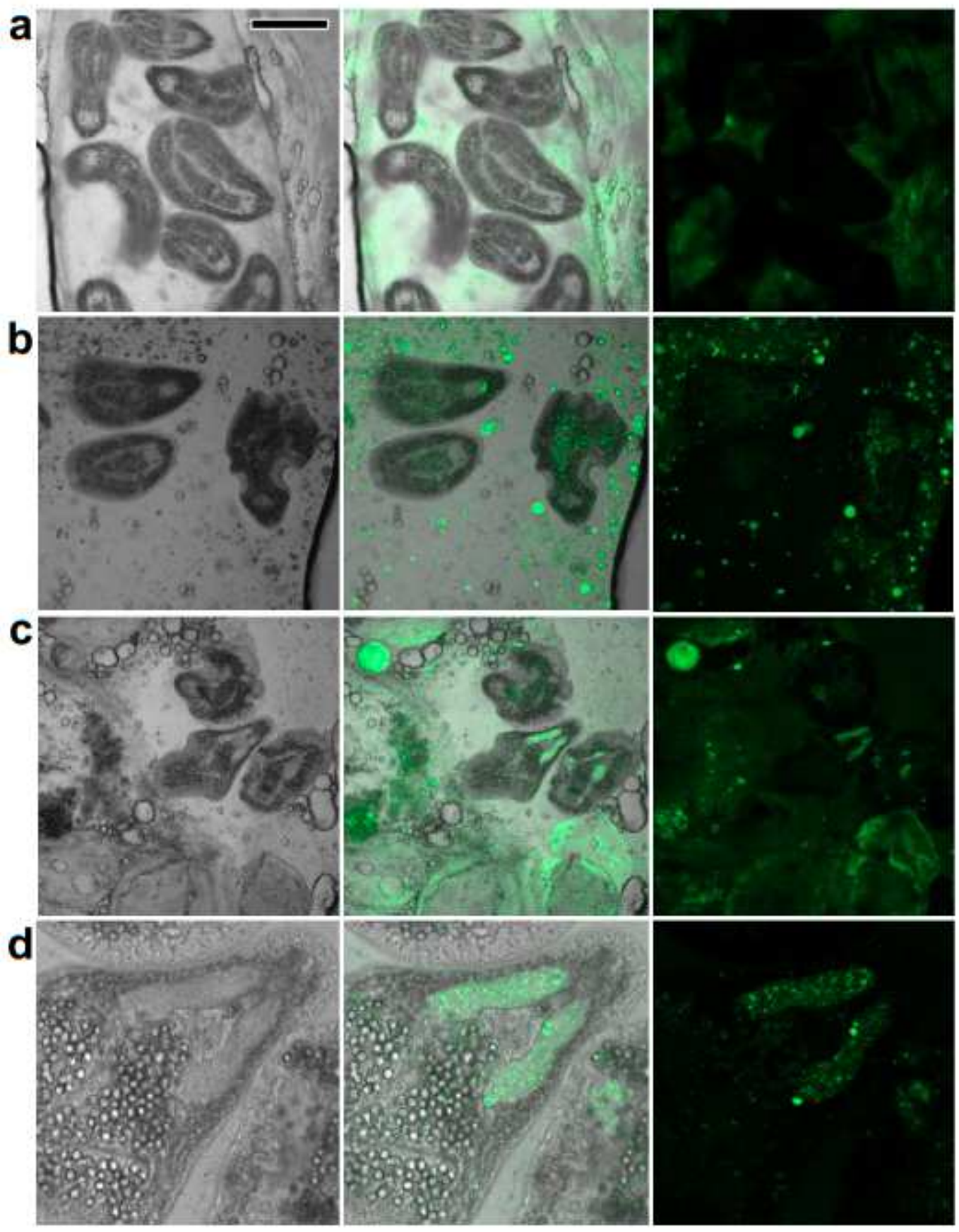

Figure 7

Confocal laser scanning micrographs (phase contrast, merge and epifluorescence of fluorescein isothiocyanate) of digenetic trematodes in intestines of Corydoras schwartzi after $1 \mathrm{~h} \mathrm{(a),6} \mathrm{h} \mathrm{(b)} \mathrm{and} 24$ $\mathrm{h}$ (c) of oral administration of fluorescently labeled chitosan-N-arginine and alginate bioparticles containing $38 \mu \mathrm{M}$ praziquantel. The amplification (d) evidences the presence of bioparticles in internal organs of a parasite from the picture above. Scale bar expands $500 \mu \mathrm{m}$. 


\section{Figure 8.}
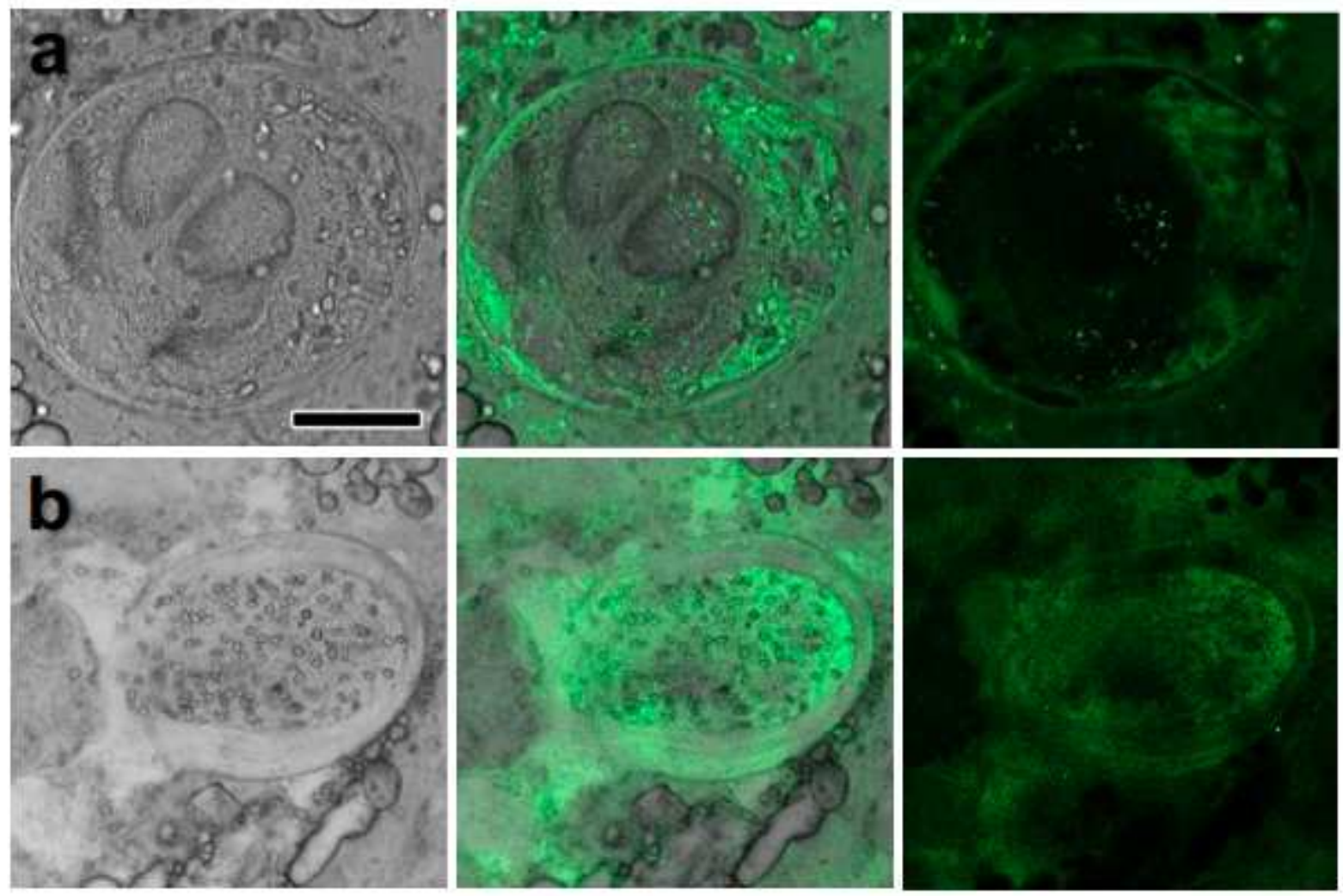

\section{Figure 8}

Confocal laser scanning micrographs (phase contrast, merge and epifluorescence of fluorescein isothiocyanate) of cysts of digenetic trematodes in intestines of Corydoras schwartzi after $6 \mathrm{~h}$ of oral administration of fluorescently labeled chitosan- $\mathrm{N}$-arginine and alginate bioparticles containing $38 \mu \mathrm{M}$ praziquantel. At least two metacercariae are focused inside the cyst in (a) while a cyst containing early stage metacercariae is burst on its left side in (b). Scale bar expands $500 \mu \mathrm{m}$ for all. 


\section{Figure 9.}

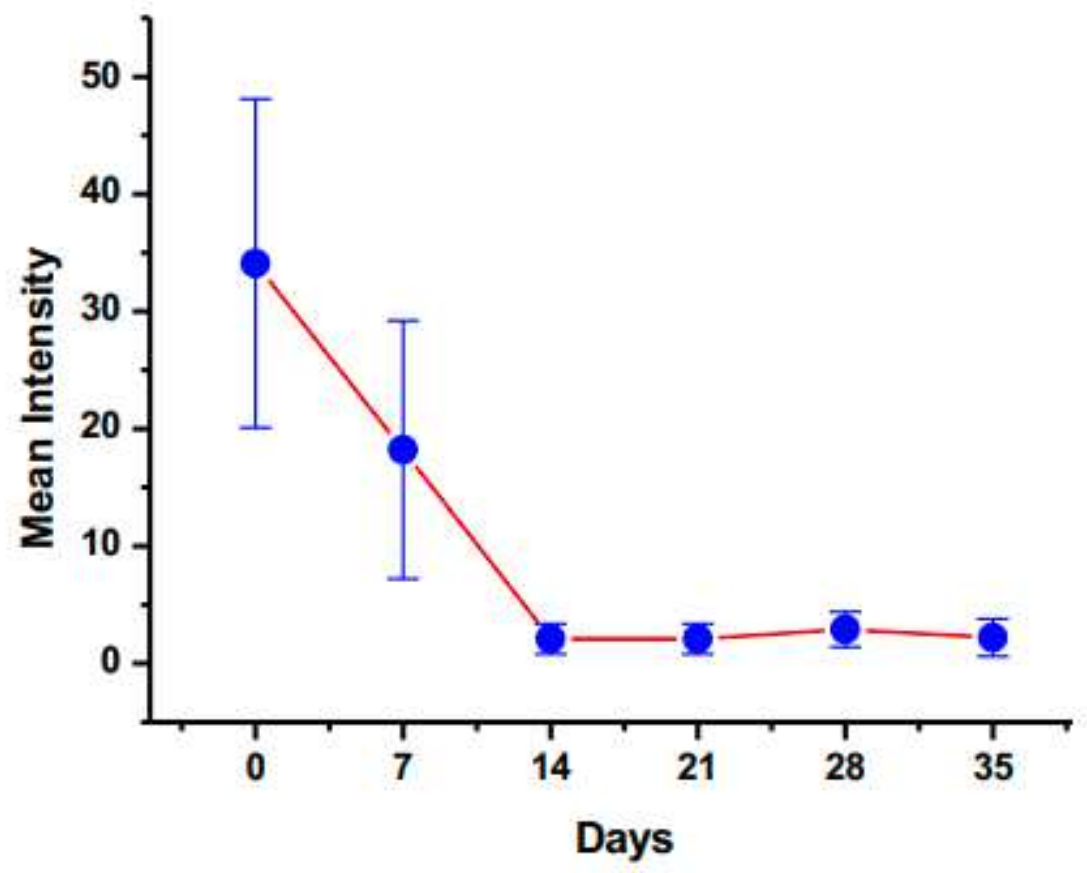

Figure 9

Mean intensity (MI) of intestinal parasites found in Corydoras fish during 35 days after the treatment with oral administration of praziquantel-bioparticles. 


\section{Figure 10.}

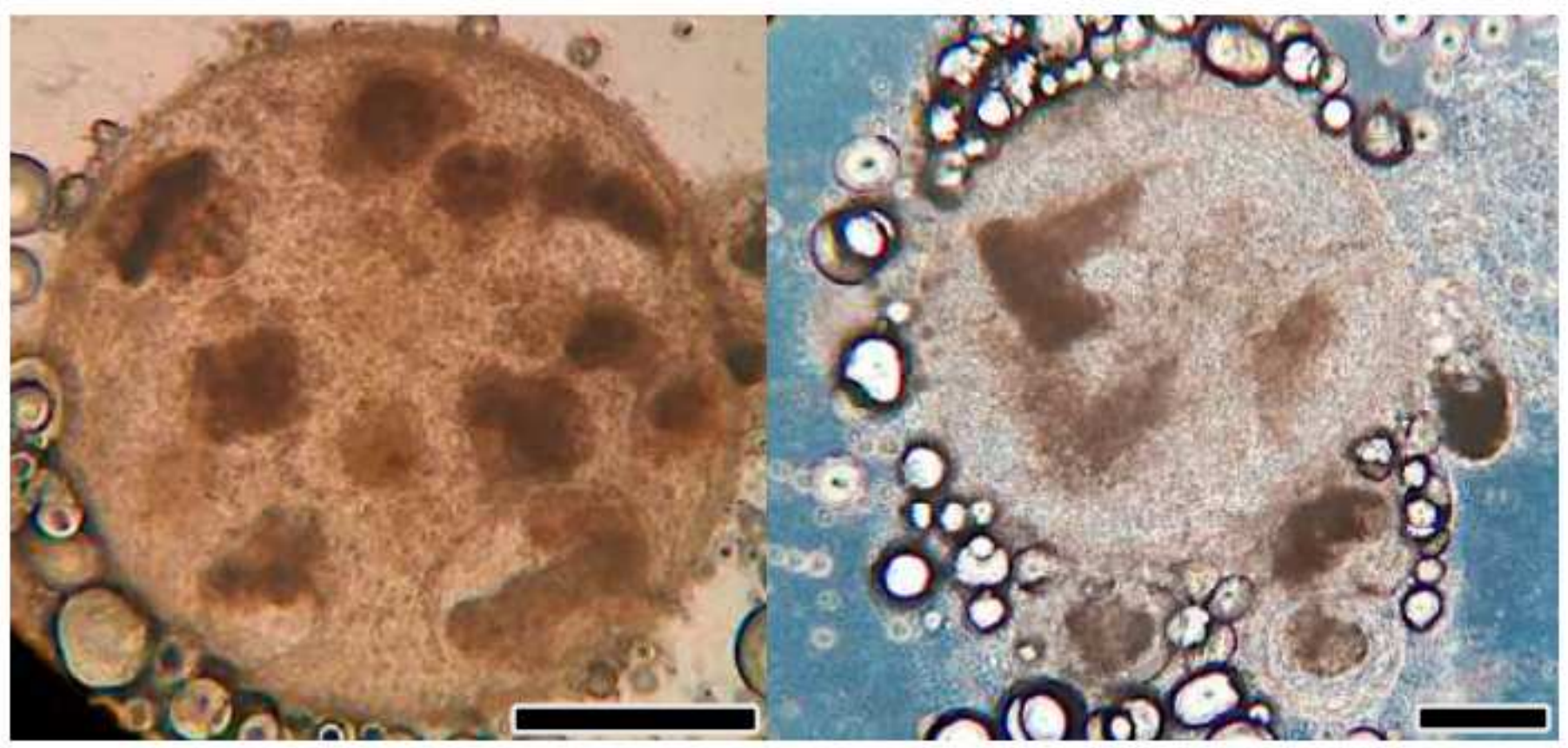

Figure 10

Digenetic trematode cysts found in intestine of Corydoras schwartzi containing parasites in advanced state of decomposition after one week of praziquantel-bioparticles administration. Scale bars expand $500 \mu \mathrm{m}$

\section{Supplementary Files}

This is a list of supplementary files associated with this preprint. Click to download.

- Table1.png

- Table2.png

- GraphicalAbstract.pdf 